\title{
Nonmonotonic Magnetic Susceptibility of Dipolar Hard-Spheres at Low Temperature and Density
}

\author{
Sofia Kantorovich, ${ }^{1,2}$ Alexey O. Ivanov, ${ }^{1}$ Lorenzo Rovigatti, ${ }^{2}$ José Maria Tavares, ${ }^{3,4}$ and Francesco Sciortino ${ }^{2,5}$ \\ ${ }^{1}$ Ural Federal University, Lenin Avenue 51, 620083, Ekaterinburg, Russia \\ ${ }^{2}$ Dipartimento di Fisica, Università di Roma La Sapienza, Piazzale Aldo Moro 2, 00185 Roma, Italy \\ ${ }^{3}$ Instituto Superior de Engenharia de Lisboa-ISEL, Rua Conselheiro Emídio Navarro 1, P-1950-062 Lisbon, Portugal \\ ${ }^{4}$ Centro de Física Teórica e Computacional, Avenida Professor Gama Pinto 2, P-1649-003 Lisbon, Portugal \\ ${ }^{5}$ CNR-ISC, Università di Roma La Sapienza, Piazzale A. Moro 2, 00185 Roma, Italy
}

(Received 23 December 2012; published 5 April 2013)

\begin{abstract}
We investigate, via numerical simulations, mean field, and density functional theories, the magnetic response of a dipolar hard sphere fluid at low temperatures and densities, in the region of strong association. The proposed parameter-free theory is able to capture both the density and temperature dependence of the ring-chain equilibrium and the contribution to the susceptibility of a chain of generic length. The theory predicts a nonmonotonic temperature dependence of the initial (zero field) magnetic susceptibility, arising from the competition between magnetically inert particle rings and magnetically active chains. Monte Carlo simulation results closely agree with the theoretical findings.
\end{abstract}

DOI: 10.1103/PhysRevLett.110.148306

PACS numbers: 82.70.-y, 61.20.Gy, 61.20.Ja, 75.50.Mm

More than 30 years have passed since the first experimental discovery of a nonmonotonic temperature $(T)$ dependence of the initial (zero-field) magnetic susceptibility $\chi$ in magnetic fluids [1]. The observation of a maximum in $\chi$ on cooling was immediately followed by controversial discussions among experimentalists and theorists [2-8]. It was debated whether the maximum was an equilibrium property of all ferrofluids or the fluid carrier was becoming so viscous that the rotational degrees of freedom of particles were blocked with respect to the accessible frequencies of the probing field [2,4]. Experiments were not able to provide evidence of the formation of magnetically inert aggregates of particles which could explain the low $T$ decrease of susceptibility. At the same time, the interesting hypothesis of a dynamic arrest of the magnetic particles themselves, due to the formation of a dipolar glass [1,9], did not succeed in providing a coherent picture of the ferrofluid behavior at low $T$.

The debate on the nonmonotonic behavior of $\chi$ has remained silent for almost 10 years. Recently, Lebedev [7] successfully synthesized a magnetic fluid, whose carrier remained liquid down to $160 \mathrm{~K}$, and measured the initial susceptibility at frequencies down to $0.01 \mathrm{~Hz}$, providing definitive evidence of the $\chi$ maximum in thermal equilibrium and calling for a renewed effort in the direction of understanding the physical origin of such a phenomenon.

At the same time, several simulation studies [10-17] of dipolar particles have attempted to elucidate the phase behavior and the microscopic structural crossovers [12,18-26] which take place in ferrofluids. Indeed, the dipolar interaction has a strong directional component favoring the head-to-tail arrangement of the particles and the formation, at low $T$, of linear aggregates. Some of these studies showed the presence of ringlike structures in addition to the expected linear chains, a finding confirmed also by recent experimental evidence [27]. Numerical investigations of the paradigmatic dipolar hard-sphere (DHS) model-monodisperse hard spheres of diameter $d$, possessing a point dipole moment $m$ in their centershighlighted the importance of the competition between rings and chains in the low-density low- $T$ regime $[28,29]$. Similar results for patchy colloids were obtained in Ref. [30].

In this Letter, we present a novel theoretical analysis of the behavior of the initial susceptibility. Our study is motivated by the availability of accurate simulation data, providing for the first time access to the strong coupling regime in equilibrium, as well as by the evidence of the ring-chain structural crossovers taking place at low $T$. Specifically, we develop a parameter-free combined mean field and density functional theory for the chainring equilibrium at low densities in DHSs and a parameter-free model for the susceptibility of chains and rings of fixed size. We then combine these two results to evaluate $\chi(T)$ at low densities. We test the theoretical predictions, step by step, with previous and novel Monte Carlo results. Both theory and simulations reveal the existence of a maximum in $\chi(T)$. The agreement between theory and simulations is remarkable at all steps and provides final evidence that the $\chi$ maximum originates from the appearance of magnetically inert aggregates (the rings) leading to an effective reduction of the magnetically responsive fraction of particles. This mechanism comes into play when both the temperature and the density are very low.

Numerical methods.-We employ Monte Carlo simulations in the canonical ensemble to investigate dilute DHS 
systems with volume fractions $\varphi=N v / V \ll 1$, where $N=5000$ is the number of particles, $v \equiv \pi d^{3} / 6$ is the volume of a particle, and $V$ is the volume of the system. For simplicity, we set $d=1, m=1$, and Boltzmann constant $k_{B}=1$. The implementation of advanced volume-bias techniques [28,31] allows us to equilibrate the system down to $T^{*}=0.125$ (in units of $m^{2} / d^{3}$ ). To accurately take into account dipole-dipole interactions we used Ewald summation. The total dipole moment auto-correlation function was used to check statistical independence of measurements. It allowed us to reach error bars on the order of symbol size in all figures. For further details on the simulation approach, see Ref. [28]. To partition particles into clusters, we employ a mixed distance-energy criterion: two particles are considered as bonded if their interaction energy is negative and if their relative distance is smaller than $r_{b}=1.3$ [29]. A chain contains two singlebonded particles connected by particles having two neighbors. If all particles in a cluster have only two neighbors then the cluster is labelled as a ring. Any other kind of aggregates is labelled as a branched cluster. The fraction of particles in branched structures is negligible in the investigated volume fraction range.

Theory.-The key hypothesis of our work is the assumption that the decrease of $\chi$ at low $T$ arises from the progressive thermodynamic stabilization of the ring structures, whose magnetic response we consider to be negligible. We foresee a progressive evolution of $\chi$ on cooling which starts from the independent particles value at high $T$, and increases anomalously due to the formation of linear chains at smaller $T$ to pass through a maximum when the equilibrium between chains and rings starts to favor closed structures. We start by developing a theoretical approach to model the density and $T$ dependence of the ratio of particles in rings and chains, appropriate for the case of low densities. We write the free energy density for an ideal mixture of dipolar chains and rings as [30,32-34]

$$
\frac{F\left[\left\{g_{n}\right\},\left\{f_{n}\right\}\right]}{V k_{B} T}=\sum_{n=1}^{\infty} g_{n} \ln \frac{g_{n} v}{e Q_{n}}+\sum_{n=5}^{\infty} f_{n} \ln \frac{f_{n} v}{e W_{n}},
$$

where $g_{n}$ and $f_{n}$ are the equilibrium volume fractions of chains and rings, respectively; $Q_{n}$ and $W_{n}$ denote the corresponding (normalized by $V / v$ ) partition functions of an $n$-particle chain and ring. The free-energy functional [Eq. (1)] has to be minimized with respect to the distributions $\left\{g_{n}\right\}$ and $\left\{f_{n}\right\}$ preserving $\varphi$,

$$
\sum_{n=1}^{\infty} g_{n} n+\sum_{n=5}^{\infty} f_{n} n=\frac{\varphi}{v} .
$$

Guided by ground state calculations [35] and numerical results $[28,29,36]$, we assume that rings smaller than five particles do not form, so that the ring contribution in Eq. (2) starts from $n=5$. For low temperature, a simple nearest-neighbor approach would fail to describe the long-range magnetic dipole-dipole interaction between the particles belonging to one cluster. As discussed in detail in the Supplemental Material [37], the partition functions of a chain and of a ring can be approximated as

$$
Q_{n}\left(T^{*}\right)=q^{C(n)} ; \quad W_{n}\left(T^{*}\right)=Q_{n}\left(T^{*}\right) \frac{q^{R(n)-C(n)}}{n^{3 \nu+1}},
$$

where

$$
\begin{aligned}
& R(n)=\frac{n}{2} \sin ^{3} \frac{\pi}{n}\left(\sum_{k=1}^{[(n-1) / 2]} \frac{\cos ^{2}\left(\frac{\pi k}{n}\right)+1}{\sin ^{3}\left(\frac{\pi k}{n}\right)}+\mathrm{R}_{(n+1) / 2}\right) ; \\
& C(n)=\sum_{k=1}^{n} \frac{n-k}{k^{3}} \sim n \zeta(3)-\frac{\pi^{2}}{6}, \quad(n \geq 4),
\end{aligned}
$$

with $\zeta(3)$ denoting the Riemann zeta function of three; $\mathbf{R}_{(n+1) / 2}$ stands for the residual of division, and [·] has the meaning of the integer part of the expression in the brackets. The low- $T$ dimer partition function $q$ (note that $C(2)=1$ and hence $\left.Q_{2}\left(T^{*}\right)=q\right)$, derived first by de Gennes and Pincus [38], is

$$
q\left(T^{*}\right)=\frac{T^{* 3}}{3} \exp \left(\frac{2}{T^{*}}\right) .
$$

In Eq. (3), $\nu=0.588$ is the self-avoiding random walk exponent. The term $1 / n^{3 \nu+1}$ in $W_{n}\left(T^{*}\right)$ captures the difference in entropy between chains and rings arising from the $n$ ways of opening a ring to form a chain; the difference between the numbers of self-avoiding paths of chains and rings is proportional to $n^{3 \nu}$ [39]. Finally, minimizing Eq. (1), one obtains compact expressions for $g_{n}$ and $f_{n}$,

$$
g_{n}=\frac{1}{v} Q_{n} p^{n}, \quad f_{n}=\frac{1}{v} W_{n} p^{n} .
$$

Here, $p$, the Lagrange multiplier to be found from Eq. (2), has the meaning of activity. Figure 1 shows the resulting (parameter-free) prediction for the fractions of particles aggregated in rings and in chains and compares them to corresponding Monte Carlo (MC) results. The redistribution of particles between chains and rings becomes vivid. For all investigated volume fractions, once $T^{*} \sim 0.12$, an almost complete crossover from chains to rings takes place.

In order to estimate the ability of the theory to predict the cluster size distribution of rings and chains at different $T^{*}$, we plot in Fig. 2 the ratio $f_{n} / g_{n}$ as a function of $n$. For values of the ratio $f_{n} / g_{n}$ greater (smaller) than 1 , rings are more (less) abundant than chains of the same size. The ratio $f_{n} / g_{n}$ coincides with $W_{n} / Q_{n}$ [see Eq. (6)] showing that the equilibrium between chains and rings is controlled by the subtle interplay between the energetic gain of forming one additional bond and by the entropic penalty of joining the two chain ends when converting a chain into a ring. The numerical results for this ratio, displayed in Fig. 2, show a small dependence on density at fixed temperature (for the low densities simulated), supporting 


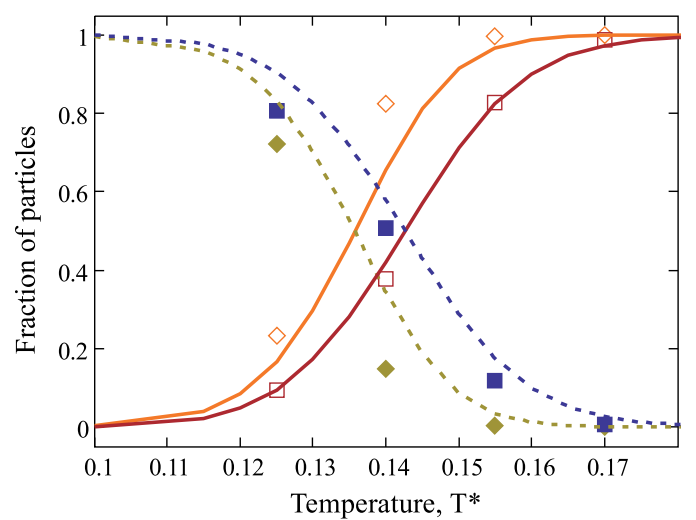

FIG. 1 (color online). Fractions of particles aggregated in rings and chains as a function of $T^{*}$ for two different densities. Simulation data are presented with symbols: open squares, fraction of particles in chains; filled squares, fraction of particles in rings $\left(\varphi=2.62 \times 10^{-4}\right)$; open diamonds, chains; and filled diamonds, rings $\left(\varphi=5.24 \times 10^{-5}\right)$. Theoretical results for the same densities are plotted with lines: solid lines, chains; and dashed lines, rings. In both cases, there is an evident redistribution of particles from chains to rings in the narrow range $0.13<$ $T^{*}<0.15$. In the simulations, a small fraction of branched structures is detected, and, hence, the sum of the fraction of particles in chains and rings does not always sum up to one.

the density independence of the ratio $W_{n} / Q_{n}$ assumed in the theory. Both $\mathrm{MC}$ results and theoretical predictions show that for $T^{*}>0.155, f_{n} / g_{n}$ remains smaller than unity for all $n$. For $T^{*}<0.155$, on the other hand, the number of intermediately sized rings becomes larger than

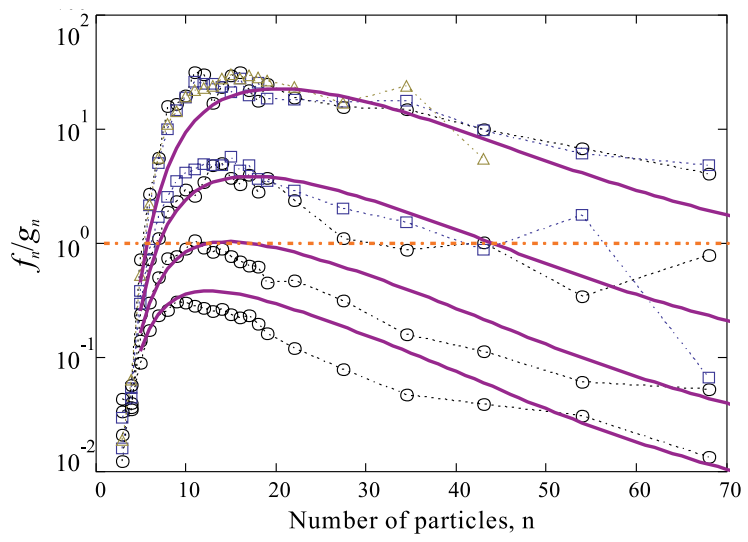

FIG. 2 (color online). Ratio $f_{n} / g_{n}$ as a function of $n$ at different $T^{*}$ and $\varphi$. Log scale is used along the ordinate axis. From the top to the bottom the reduced temperature increases: $T^{*}=$ $0.125 ; 0.140 ; 0.155 ; 0.170$. Theoretical results of Eq. (6) are plotted with solid lines and are $\varphi$ independent. Simulation data are presented with symbols: circles, $\varphi=3.67 \times 10^{-3}$; squares, $\varphi=2.62 \times 10^{-4}$; and triangles, $\varphi=3.67 \times 10^{-5}$. For $T^{*}=$ $0.155,0.170$ the amount of rings observed in simulations is not sufficient to provide reliable statistics. The rings are more probable than chains of a corresponding size above the horizontal dashed-dotted line $f_{n} / g_{n}=1$. that of chains. The position of the maximum $\left(n_{\max }\right)$ of the ratio $f_{n} / g_{n}$ depends on $T^{*}$ and it can be written as $n_{\max }\left(T^{*}\right)=2 \pi \ln q\left(T^{*}\right) /(3 \nu+1)$. We find $10<n_{\max }<18$, when $0.125<T^{*}<0.17$, in good agreement with simulation data. The second step in our study is the formulation of a parameter-free model for the susceptibility of the system as a sum over the contributions of chains and rings of fixed size $n$. We assume that the susceptibility of rings is always zero, due to the fact that their average total dipole moment vanishes. Simulation data provide a confirmation that the magnetic response of a ring is several orders of magnitude smaller than that of a chain of the same size.

We write the total initial magnetic susceptibility of the low density DHS restricting only to the chains contribution as [40]

$$
\begin{gathered}
\chi\left(T^{*}\right)=\frac{8 v}{T^{*}}\left(1+\frac{\chi_{L}^{C}}{3}\right) \sum_{n=1}^{\infty} g_{n}\left\langle m_{n}^{2}\right\rangle, \\
\left\langle m_{n}^{2}\right\rangle=n+2 \frac{K}{(1-K)^{2}}\left(n-1+K^{n}-n K\right) .
\end{gathered}
$$

Here, $\chi_{L}^{C}=8 \varphi_{C} / T^{*}$ is the Langevin susceptibility of all particles aggregated in chains, whose volume fraction is $\varphi_{C}$, at $T^{*}$. The term $\left(1+\frac{\chi_{L}^{C}}{3}\right)$ is the mean field contribution modeling long range interactions (for the derivation, see Ref. [41]). The final sum contains the chain contribution, with $\left\langle m_{n}^{2}\right\rangle$ having the meaning of a dimensionless meansquared dipole moment of a chain made of $n$ DHS particles. The expression for $\left\langle m_{n}^{2}\right\rangle$ in Eq. (8) has been derived in Ref. [40]. For DHS particles, the dipolar correlation coefficient $K$ assumes the simple form

$$
K\left(T^{*}\right)=\operatorname{coth}\left(1 / 2 T^{*}\right)-2 T^{*} .
$$

Equation (7) assumes that $\chi$ can be written as a sum over the contributions to the susceptibility of each chain of size $n$,

$$
\chi_{c}\left(n, T^{*}\right)=8\left(1+\frac{\chi_{L}^{C}}{3}\right)\left\langle m_{n}^{2}\right\rangle / T^{*} .
$$

Once more, simulations can be utilized to provide a close comparison, at the single chain level, of the quality of the theoretical predictions. We, thus, partition particles into clusters and select all chains according to their length. The corresponding value of $\chi_{c}\left(n, T^{*}\right)$ is then evaluated averaging over all chains with the same $n$ by evaluating the fluctuation-dissipation relationship

$$
\chi_{c}\left(n, T^{*}\right)=\frac{4 \pi}{3} \frac{d^{3}}{V T^{*}}\left(\left\langle M_{n}^{2}\right\rangle-\left\langle M_{n}\right\rangle^{2}\right),
$$

where $M_{n}$ is the dimensionless total dipole moment of an $n$-particle chain, and \langle\rangle indicates an average over all chains with the same length $n$

The results, plotted in Fig. 3, demonstrate a very good agreement between theory and simulation even at the level 


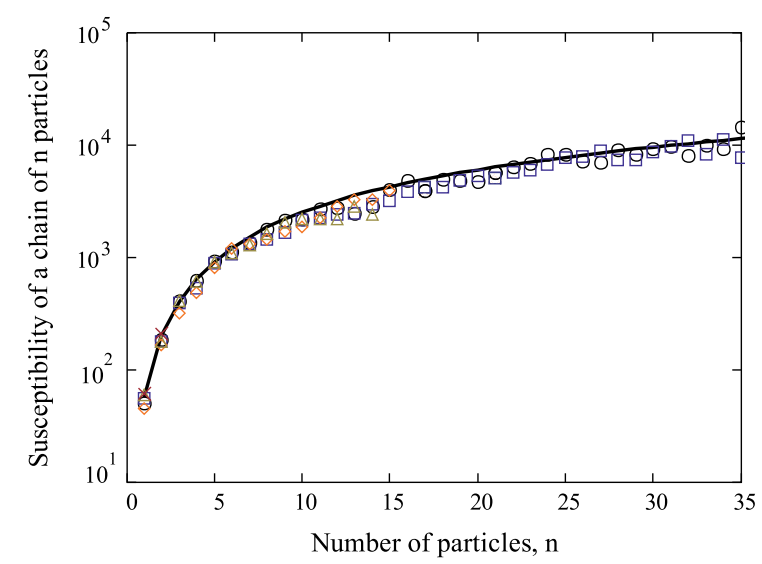

FIG. 3 (color online). Initial magnetic susceptibility of a single chain composed of $n$ particles at $T^{*}=0.155$. Log scale is used along the ordinate axis. Theoretical result of Eq. (10) is plotted with the solid line. Simulation data are presented with symbols: circles, $\varphi=3.67 \times 10^{-3} ; \quad$ squares, $\varphi=2.62 \times 10^{-4}$; diamonds, $\varphi=5.24 \times 10^{-5} ;$ triangles, $\varphi=3.67 \times 10^{-5}$; and crosses, $\varphi=5.24 \times 10^{-6}$.

of a single chain. It is worth noting that the magnetic response of an $n$-particle chain is the same for all chains of the same size, independently from the volume fraction employed in the simulation. It means that the intercluster correlations play a negligible part for the parameters studied here. This validates our theoretical assumption to model the system as a chain-ring ideal mixture, Eq. (1). Finally, we compare the theoretical prediction for the initial susceptibility of the DHS systems with the analogous quantity computed from the MC configurations, evaluated from the fluctuation-dissipation relationship. The comparison is reported in Fig. 4. For high temperature, $\chi$ obeys a Curie-type $1 / T^{*}$ law and, depending on particle density, might be well described by the modified meanfield approach [41]. In Fig. 4, the values of $\varphi$ are so low that even the Langevin one-particle model with the initial susceptibility $\chi_{L}=8 \varphi / T^{*}$ appears to be sufficient. However, in Fig. 4, both MC and theoretical results show that $\chi$ has a maximum for $0.12<T^{*}<0.15$, after which the curves rapidly decrease for all densities. The decrease is so strong, that the initial susceptibility becomes lower than $\chi_{L}$. Dashed lines in Fig. 4 show how strong the increase of theoretically predicted initial magnetic susceptibility could have been, if only chains had formed in the system.

Conclusions.- Our study demonstrates that the nonmonotonic temperature dependence of $\chi$ in the low-density DHS system is indeed triggered by ring formation. The drastic effect is caused by the exclusion of a significant fraction of particles from the collective magnetic response. One might consider the excluded particles to form "magnetic holes" in the system and as such to reduce the density of "active" magnetic dipoles. In the specific

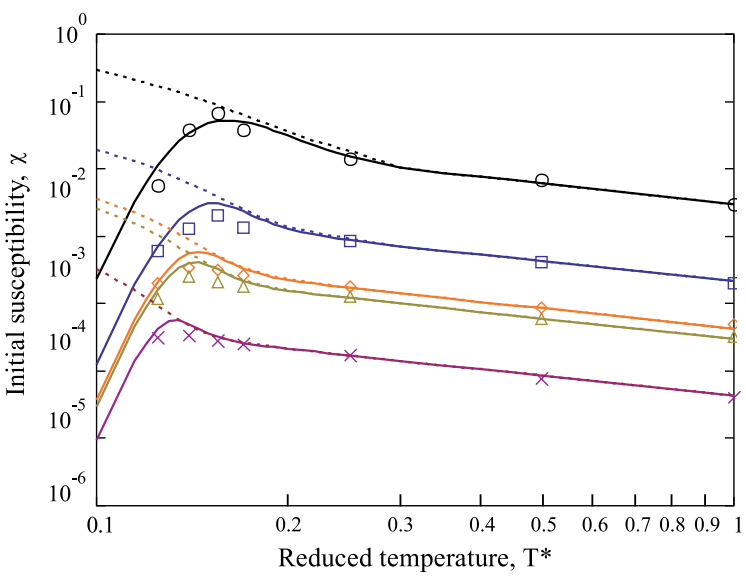

FIG. 4 (color online). Initial magnetic susceptibility as a function of reduced temperature $T^{*}$ for various volume fractions. Log scale is used along both axes. Theoretical results of Eq. (7) are plotted with solid lines. Simulation data are presented with symbols: circles, $\varphi=3.67 \times 10^{-3}$; squares, $\varphi=2.62 \times 10^{-4}$; diamonds, $\varphi=5.24 \times 10^{-5}$; triangles, $\varphi=3.67 \times 10^{-5}$; and crosses, $\varphi=5.24 \times 10^{-6}$. In all cases, there is a maximum of $\chi$, which slightly shifts towards lower $T^{*}$ with decreasing $\varphi$. Dashed lines: initial magnetic susceptibility for the same range of parameters obtained in theory assuming that only chains (no rings) can form in the system.

case of low-density DHSs the magnetic hole is a ring made of dipolar particles, as the average magnetic moment of a ring is zero. The appearance of rings leads to an effective reduction of the magnetically responsive fraction of particles. This mechanism comes into play when both the temperature and the density are very low. This explains why, in previous studies of macroscopic properties, the signature of ring formation in DHS systems has not been observed [10,12,15,18,20,21]. For higher densities, the microstructure of the DHS system becomes more complex and simulation studies [28,29] have shown the presence of branched structures which span the entire system. This "gelation"-type crossover, giving rise to structures which could also be characterized by closed magnetic dipole moments, might also lead to the aforementioned effective exclusion of particles and result in the drop of the magnetic susceptibility, also at large densities. We are confident that the presented theoretical predictions will motivate further experiments at low densities and temperatures (at which the carrier might still be liquid $[7,8]$ ).

This research is supported by FP7-IDEAS-ERC226207-PATCHYCOLLOIDS and ITN-234810COMPLOIDS. S. K. is grateful to RFBR Grants No. mol-a 1202-31-374 and No. mol-a-ved 12-02-33106. S. K. and A.I. are supported by the Ministry of Science and Education of RF 2.609.2011. A. I. thanks Sapienza for hospitality. J. M. T. acknowledges financial support from the Portuguese Foundation for Science and Technology (FCT) under Contracts No. PEst-OE/FIS/UI0618/2011 and No. PTDC/FIS/098254/2008. 
[1] A. Tari, J. Popplewell, and S. Charles, J. Magn. Magn. Mater. 15, 1125 (1980).

[2] Y. Dikanskii, Magnetohydrodynamics 18, 237 (1982).

[3] S. Menear, A. Bradbury, and R. Chantrell, J. Magn. Magn. Mater. 43, 166 (1984).

[4] M. Shliomis, A. Pshenichnikov, K. Morozov, and I. Shurubor, J. Magn. Magn. Mater. 85, 40 (1990).

[5] M. Holmes, K. O'Grady, and J. Popplewell, J. Magn. Magn. Mater. 85, 47 (1990).

[6] A. F. Pshenichnikov and A. V. Lebedev, J. Chem. Phys. 121, 5455 (2004).

[7] A. Lebedev, Colloid J. 72, 815 (2010).

[8] A. Lebedev and S. Lysenko, J. Magn. Magn. Mater. 323, 1198 (2011).

[9] A. Minakov, I. Zaitsev, and U. Lesnih, J. Magn. Magn. Mater. 85, 60 (1990).

[10] A.-P. Hynninen and M. Dijkstra, Phys. Rev. Lett. 94, 138303 (2005).

[11] R. van Roij, Phys. Rev. Lett. 76, 3348 (1996).

[12] Y. Levin, Phys. Rev. Lett. 83, 1159 (1999).

[13] A. Yetheraj and A. van Blaaderen, Nature (London) 421, 513 (2003).

[14] A. K. Agarwal and A. Yethiraj, Phys. Rev. Lett. 102, 198301 (2009).

[15] J. Richardi and J.-J. Weis, J. Chem. Phys. 135, 124502 (2011).

[16] J. Russo, J. M. Tavares, P. I. C. Teixeira, M. M. Telo da Gama, and F. Sciortino, Phys. Rev. Lett. 106, 085703 (2011).

[17] J. M. Tavares, J. J. Weis, and M. M. Telo da Gama, Phys. Rev. E 65, 061201 (2002).

[18] J. J. Weis and D. Levesque, Phys. Rev. Lett. 71, 2729 (1993).

[19] S. Klapp and F. Forstmann, J. Chem. Phys. 106, 9742 (1997).

[20] P. J. Camp, J. C. Shelley, and G. N. Patey, Phys. Rev. Lett. 84, 115 (2000).

[21] P. J. Camp and G. N. Patey, Phys. Rev. E 62, 5403 (2000).

[22] P. D. Duncan and P. J. Camp, Phys. Rev. Lett. 97, 107202 (2006).
[23] G. Ganzenmuller and P. J. Camp, J. Chem. Phys. 126, 191104 (2007).

[24] J. Jordanovic and S. H. L. Klapp, Phys. Rev. Lett. 101, 038302 (2008).

[25] A. O. Ivanov, S. S. Kantorovich, and P. J. Camp, Phys. Rev. E 77, 013501 (2008).

[26] G. Ganzenmueller, G. Patey, and P. Camp, Mol. Phys. 107, 403 (2009).

[27] M. Klokkenburg, R. P. A. Dullens, W. K. Kegel, B. H. Erné, and A. P. Philipse, Phys. Rev. Lett. 96, 037203 (2006).

[28] L. Rovigatti, J. Russo, and F. Sciortino, Phys. Rev. Lett. 107, 237801 (2011).

[29] L. Rovigatti, J. Russo, and F. Sciortino, Soft Matter 8, 6310 (2012).

[30] J. M. Tavares, L. Rovigatti, and F. Sciortino, J. Chem. Phys. 137, 044901 (2012).

[31] B. Chen and J. I. Siepmann, J. Phys. Chem. B 105, 11275 (2001).

[32] R. P. Sear and G. Jackson, Phys. Rev. E 50, 386 (1994).

[33] A. Avlund, G. Kontogeorgis, and W. Chapman, Mol. Phys. 109, 1759 (2011).

[34] A. Galindo, S. Burton, G. Jackson, D. Visco, and D. A. Kofke, Mol. Phys. 100, 2241 (2002).

[35] T. A. Prokopieva, V.A. Danilov, S.S. Kantorovich, and C. Holm, Phys. Rev. E 80, 031404 (2009).

[36] S. Kantorovich, J. J. Cerdà, and C. Holm, Phys. Chem. Chem. Phys. 10, 1883 (2008).

[37] See Supplemental Material at http://link.aps.org/ supplemental/10.1103/PhysRevLett.110.148306 for details on how to calculate chain and ring partition functions; we also thoroughly discuss all the approximations for the shape of chains and rings made in the present Letter.

[38] P. de Gennes and P. Pincus, Z. Phys. B 11, 189 (1970).

[39] J. P. Wittmer, P. van der Schoot, A. Milchev, and J. L. Barrat, J. Chem. Phys. 113, 6992 (2000).

[40] V. S. Mendelev and A. O. Ivanov, Phys. Rev. E 70, 051502 (2004).

[41] A. O. Ivanov and O. B. Kuznetsova, Phys. Rev. E 64, 041405 (2001). 